Hydrology and Earth System Sciences, 5(4), 645-651 (2001) C $\quad$ EGS

\title{
Rainfall dynamics at different temporal scales: A chaotic perspective
}

\author{
B. Sivakumar \\ Department of Land, Air \& Water Resources, University of California, Davis, CA 95616, USA \\ E-mail: sbellie@ucdavis.edu
}

\begin{abstract}
This study of the behaviour of rainfall dynamics at different temporal scales identifies the type of approach most suitable for transformation of rainfall data from one scale to another. Rainfall data of four different temporal scales, i.e. daily, 2-day, 4-day and 8-day, observed over a period of about 25 years at the Leaf River basin, Mississippi, USA, are analysed. The correlation dimension method is employed to identify the behaviour of rainfall dynamics. The finite correlation dimensions obtained for the four rainfall series $(4.82,5.26,6.42$ and 8.87 , respectively) indicate the possible existence of chaotic behaviour in the rainfall observed at the four scales. A possible implication of this might be that the rainfall processes at these scales are related through a chaotic (scale-invariant) behaviour. However, a comparison of the correlation dimension and coefficient of variation of each of the time series reveals an inverse relationship between the two (higher dimension for lower coefficient of variation and vice versa). The presence of a large number of zeros in the higher resolution time series (that could result in an underestimation of the dimension) and the possible presence of a higher level of noise in the lower resolution time series (that could result in an overestimation of the dimension) might account for such results. In view of these problems, it is concluded that the results must be verified using other chaos identification methods and the existence of chaos must be substantiated with additional evidence.
\end{abstract}

Key words: rainfall, chaos, scaling, correlation dimension, number of variables, coefficient of variation, data size, noise, zeros

\section{Introduction}

The outcomes of numerous studies of transformation of hydrological data, such as rainfall, from one scale to another, support unanimously such a possibility. Among these, the fractal (or scaling) based studies that employ multi-fractal random cascade schemes are of tremendous practical significance. An important concern of such approaches, however, is the connection between the structure of the model on the one hand, and the underlying dynamics of the process on the other. Establishing this connection is crucial. The multi-fractal random cascade approaches treat the data as a realisation of a stochastic process, whose prevalent characteristic is the multi-fractal spectrum and, therefore, may not account for the uniqueness of the data at hand. Therefore, an approach that seeks to understand the whole and unique data set, in addition to preserving the multifractal spectrum, other important qualifiers of the records, and the overall appearance of the data, is required to establish such a connection. The notion of deterministic chaos (that seemingly irregular-looking behaviour can be the result of simple deterministic systems dominantly influenced by a few nonlinear interdependent variables sensitive to initial conditions) and the related nonlinear methods of data processing have great potential.

Applications of the concept of deterministic chaos to understand hydrological processes have received considerable attention in recent times (e.g. Rodriguez-Iturbe et al., 1989; Tsonis et al., 1993; Berndtsson et al., 1994; Jayawardena and Lai, 1994; Koutsoyiannis and Pachakis, 1996; Puente and Obregon, 1996; Porporato and Ridolfi, 1997; Krasovskaia et al., 1999; Sivakumar et al., 1999a; Stehlik, 1999). That such applications have resulted in noticeable progress in the areas of identification and prediction of hydrological processes is encouraging news for hydrologists. It is believed that the concept of chaos can be employed also to solve other hydrological problems, such as data transformation as well. An attempt is made in the present study to employ the concept of chaos theory to understand the dynamics of transformation of rainfall from one scale (or resolution) to another, a problem of significant 
importance in hydrology, as the lack of high-resolution rainfall data is one of the most prominent limiting factors in hydrological calculations.

Almost all of the past studies of the existence of chaos in rainfall processes analysed time series of only one particular scale measured at the same geographical location (e.g. Berndtsson et al., 1994; Jayawardena and Lai, 1994; Sivakumar et al., 1999a) or of different scales observed in different geographical locations (e.g. Rodriguez-Iturbe et al., 1989). Hence, none could provide information about whether or not the transformation of rainfall process between different scales is chaotic. As a first step, the present study investigates rainfall series of different (temporal) scales observed at the same geographical location. Rainfall data of four different temporal scales, i.e. daily, 2-day, 4-day, and 8-day, over a period of about 25 years observed at the Leaf River basin, Mississippi, USA, are analysed (independently) to investigate the existence of chaos. The underlying assumption is that the individual behaviour of the dynamics of rainfall processes at these scales provides important information about the dynamics of the overall rainfall transformation between these scales. More specifically, if the rainfall processes at different scales exhibit chaotic behaviour, then the dynamics of the transformation between them may also be chaotic. The presence of chaos in the rainfall series is investigated by employing the correlation dimension method (e.g. Grassberger and Procaccia, 1983). The correlation dimension is a representation of the variability or irregularity of a process and furnishes information on the number of dominant variables present in the evolution of the corresponding dynamical system; it can indicate not only the existence of chaos in the rainfall process, if any, but also reveal whether the process is deterministic or stochastic, if not chaotic.

\section{Correlation dimension method}

The correlation dimension method uses the correlation integral (or function) to distinguish chaotic and stochastic systems. The Grassberger-Procaccia algorithm (Grassberger and Procaccia, 1983), employed in this study to estimate the correlation dimension of the rainfall series, uses the concept of phase-space reconstruction. For a scalar time series $X_{i}$, where $i=1,2, \ldots, N$, the phase-space can be reconstructed using the method of delays, according to (e.g. Takens, 1981):

$$
\mathbf{Y}_{j}=\left(X_{j}, X_{j+\tau}, X_{j+2 \tau}, \ldots, X_{j+(m-1) \tau}\right)
$$

where $j=1,2, \ldots ., N-(m-1) \tau / \Delta t ; m$ is the dimension of the vector $\boldsymbol{Y}_{j}$, also called the embedding dimension; and $\tau$ is a delay time. For an $m$-dimensional phase-space, the correlation function $C(r)$ is given by

$$
C(r)=\lim _{N \rightarrow \infty} \frac{2}{N(N-1)} \sum_{\substack{i, j \\(1 \leq i<j \leq N)}} H\left(r-\left|\boldsymbol{Y}_{i}-\boldsymbol{Y}_{j}\right|\right)
$$

where $H$ is the Heaviside step function, with $H(u)=1$ for $u$ $>0$, and $H(u)=0$ for $u \leq 0$, where $u=r-\left|\boldsymbol{Y}_{i}-\boldsymbol{Y}_{j}\right|, r$ is the radius of sphere centred on $\boldsymbol{Y}_{i}$ or $\boldsymbol{Y}_{j}$. If the time series is characterised by an attractor (a geometric object which characterises the long-term behaviour of a system in the phase-space) then, for positive values of $r$, the correlation function $C(r)$ is related to the radius $r$ by:

$$
\begin{aligned}
& C(r) \sim \alpha r^{v} \\
& r \rightarrow 0 \\
& N \rightarrow 0
\end{aligned}
$$

where $a$ is constant and $v$ is the correlation exponent or the slope of the $\log C(r)$ versus $\log r$ plot given by

$$
v=\lim _{\substack{r_{i} 0 æ \\ N_{i} \ngtr \\ r \rightarrow 0 \\ N \rightarrow \infty}} \frac{\log C(r)}{\log r}
$$

The slope is generally estimated by a least-squares fit of a straight line over a certain range of $r$, called the scaling region.

The presence/absence of chaos can be identified using the correlation exponent versus the embedding dimension plot. If the correlation exponent saturates and the saturation value is low, then the system is generally considered to exhibit low-dimensional chaos. The saturation value of the correlation exponent is defined as the correlation dimension of the attractor. The nearest integer above the saturation value provides the minimum number of variables necessary to model the dynamics of the attractor. On the other hand, if the correlation exponent increases without limit with increase in the embedding dimension, the system under investigation is generally considered as stochastic.

\section{Analyses, results, and discussion}

In the present study, rainfall data observed at the Leaf River basin, Mississippi, USA, are used. The climate in this region is humid subtropical, characterised by short, mild temperate winters and long, hot summers. The mean annual precipitation is about $1350 \mathrm{~mm}$. March is the wettest month with a mean rainfall of about $160 \mathrm{~mm}$, while October is driest with a mean rainfall of about $80 \mathrm{~mm}$. For this investigation, rainfall data of four different scales (or resolutions), i.e. daily, 2-day, 4-day and 8-day, observed over 
a period of about 25 years (January 1963 - December 1987) are used.

Figure 1 shows the variation of the daily rainfall series at the Leaf River basin, while Table 1 presents some of the important statistics of the above four series. The correlation functions and the exponents are now computed for the four series. The delay time, $\tau$, for the phase-space reconstruction is computed using the auto-correlation function method and is taken as the lag time at which the auto-correlation function first crosses the zero line (e.g. Holzfuss and Mayer-Kress, 1986). Figure 2 shows the variation of the auto-correlation

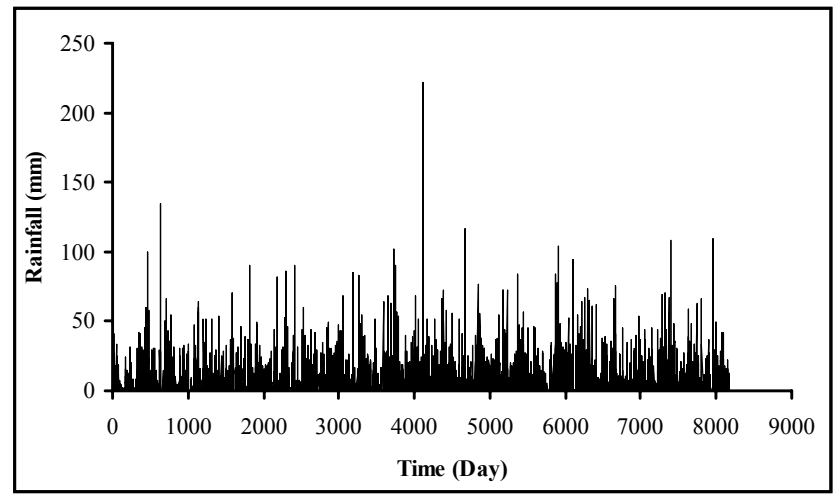

Fig. 1. Variation of daily rainfall series at the Leaf River basin

Table 1. Statistics of Leaf River rainfall data (values in $\mathrm{mm}$ )

\begin{tabular}{lrrrc}
\hline Parameter & Daily & 2-day & 4-day & 8-day \\
\hline \multirow{2}{*}{ Number of data } & 8192 & 4096 & 2048 & 1024 \\
Mean & 4.03 & 8.06 & 16.12 & 32.24 \\
Standard deviation & 10.47 & 15.61 & 22.08 & 31.90 \\
Variance & 109.46 & 243.56 & 487.62 & 1017.62 \\
Coefficient of variation & 2.60 & 1.94 & 1.37 & 0.99 \\
Maximum value & 221.52 & 221.52 & 221.52 & 234.03 \\
Minimum value & 0.00 & 0.00 & 0.00 & 0.00 \\
Number of zeros & 4467 & 1633 & 412 & 62 \\
& $(54.53 \%)$ & $(39.87 \%)$ & $(20.12 \%)$ & $(6.05 \%)$ \\
\hline
\end{tabular}

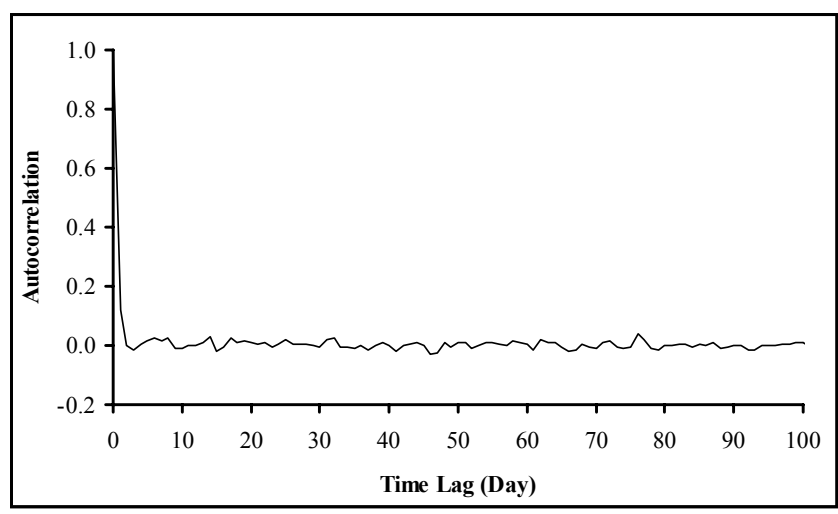

Fig. 2. Autocorrelation function for daily rainfall series
Table 2. Results of correlation dimension analysis of rainfall data

\begin{tabular}{lcccc}
\hline Parameter & Daily & 2-day & 4-day & 8-day \\
\hline Delay time & 2 & 5 & 3 & 5 \\
Correlation dimension & 4.82 & 5.26 & 6.42 & 8.87 \\
Number of variables & 5 & 6 & 7 & 9 \\
Coefficient of variation & 2.60 & 1.94 & 1.37 & 0.99 \\
Percentage of zeros & 54.53 & 39.87 & 20.12 & 6.05 \\
\hline
\end{tabular}

function against the lag time for the daily rainfall series. For the four series, the first zero value of the autocorrelation function is attained at lag times 2, 5, 3 and 5 respectively (Table 2); therefore, these values are used as the delay times in the phase-space reconstruction.

For the daily rainfall series, Fig. 3(a) shows the relationship between the correlation integral, $C(r)$, and the radius, $r$, for embedding dimensions, $m$, from 1 to 20 . The $\log C(r)$ versus $\log r$ plots indicate clear scaling regions that allow fairly accurate estimates of the correlation exponents. Figure $3(\mathrm{~b})$ presents the relationship between
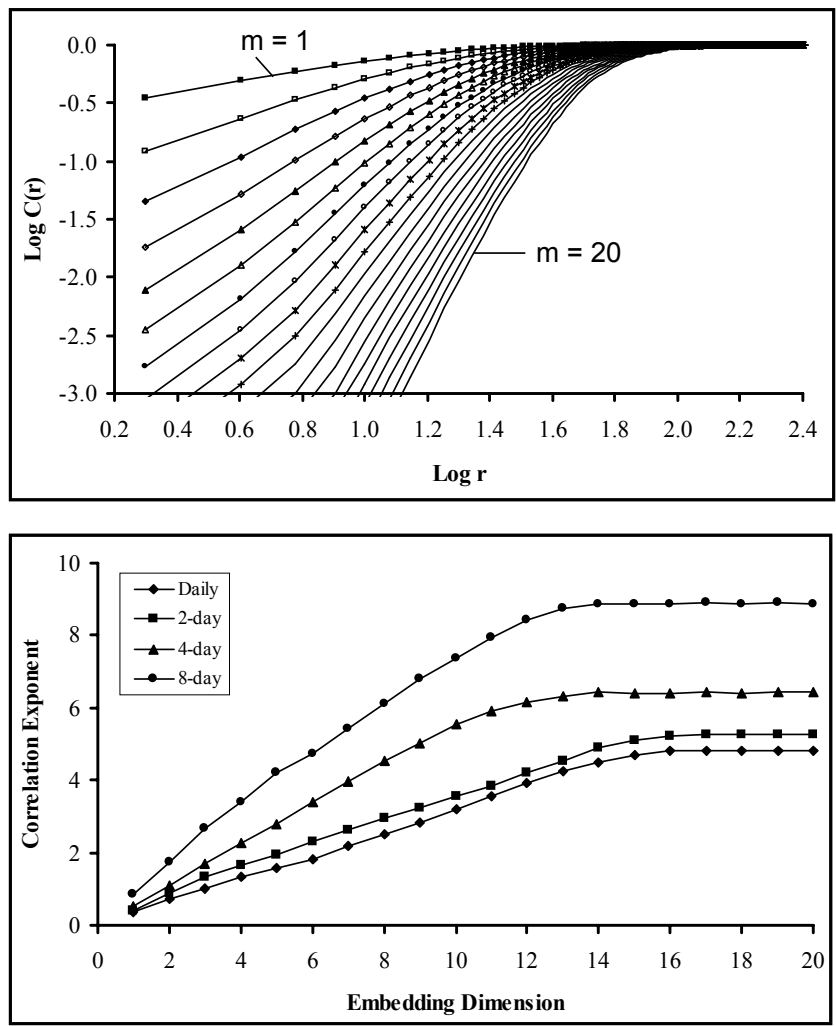

Fig. 3. (a) $\log C(r)$ versus og $r$ for daily rainfall series; and (b) Relationship between correlation exponent and embedding dimension for daily, 2-day, 4-day, and 8-day rainfall series 
the correlation exponent values and the embedding dimension values for the four rainfall series. For all the series, the correlation exponent value increases with the embedding dimension up to a certain dimension, beyond which it is saturated; this is an indication of the existence of deterministic dynamics. The saturation values of the correlation exponent (or correlation dimension) for the four rainfall series are respectively, 4.82, 5.26, 6.42 and 8.87 (Table 2). The finite correlation dimensions obtained for the four series indicate that they all exhibit chaotic behaviour. The presence of chaos at each of these four scales suggests that the dynamics of transformation of rainfall between these scales may also exhibit chaotic behaviour. This, in turn, may imply the applicability (or suitability) of a chaotic approach for transformation of rainfall data from one scale to another. However, care must be taken in adopting such an interpretation for the following reasons.

The (correlation) dimension of a time series represents the variability or irregularity of the values in the series. A series with a high variability in values provides a higher dimension, which, in turn, indicates higher complexity in the dynamics of the process. A low dimension would be the result of low variability, indicating that the dynamics of the process are less complex. The dimension results obtained above indicate that the rainfall series at the 8-day scale exhibits the highest variability. The correlation dimension value of 8.87 obtained indicates that the number of dominant variables involved in the dynamics of the 8-day rainfall series is 9 . On the other hand, the daily series, yielding a correlation dimension of 4.82, exhibits the lowest variability, indicating that the number of dominant variables involved in the daily rainfall dynamics is 5. For rainfall series at 2day and 4-day scales, the correlation dimensions obtained are respectively 5.26 and 6.42 so that the number of variables dominant in the dynamics is 6 and 7 respectively (Table 2).

The correlation dimensions and, thus, the number of variables obtained for the four rainfall series indicate that aggregating the rainfall from higher resolutions, such as daily and 2-day, to lower resolutions, such as 4-day and 8day, increases the variability of the rainfall dynamics. Such an observation seems to be contrary to what is normally observed in nature; in general, the temporal aggregation of rainfall often decreases the variability.

The values of the coefficient of variation, defined as the ratio of the standard deviation to the mean, presented in Table 1, support this latter point. The highest coefficient of variation of 2.60 is observed for the (highest resolution) daily rainfall series, indicating that the daily series exhibits the highest variability. The coefficient of variation values for the 2-day, 4-day and 8-day rainfall series are in decreasing order with values of 1.94, 1.37 and 0.99 respectively, indicating a decreasing trend in the variability, with the (lowest resolution) 8-day rainfall exhibiting the lowest variability.

The above dimension results seem to indicate that the correlation dimension method may have some serious limitations. It is not known, at this stage, whether the dimensions of the higher resolution series are underestimated or those of the lower resolution series are overestimated. Possible explanations can be provided, if either of the above is assumed correct (or wrong).

The correlation dimension method is based on the assumption that the time series is infinite and noise-free. Therefore, the application of this method to rainfall series, which are always finite and contaminated by noise (e.g. measurement error), may sometimes provide misleading results. For example, a finite and small data set may underestimate the actual dimension (e.g. Havstad and Ehlers, 1989), whereas the presence of noise may result in an overestimation of the dimension (e.g. Schreiber and Kantz, 1996).

The belief that the estimation of the correlation dimension requires a large data set is based on the assumption that the data size depends on the embedding dimension used in the phase-space reconstruction. However, Sivakumar (2000) reveals that the data size depends on the type and dimension of the underlying process, rather than the embedding dimension. The sizes of the rainfall series considered in the present study and the dimension results obtained may also be used to explain the above point. The higher resolution rainfall series, such as daily and 2-day, are significantly larger in size than that of the lower resolution series, but yield significantly lower dimensions than those of the lower resolution series. In other words, the dimension estimate increases with a decreasing data size and vice versa. This suggests that the data size could have only very little influence, if any at all, on the dimension estimate and, therefore, the chances of underestimation of the dimension for the higher resolution series (compared to the others) may be very limited. Also, it is the author's belief that the rainfall series of different scales (with a lowest scale of 8 days) observed over a period of 25 years are sufficient to understand the dynamics of the rainfall process at these scales.

Recent studies have revealed that the presence of noise in the data could result in an overestimation of the dimension, and the extent of overestimation increases with the noise level (e.g. Sivakumar et al., 1999b; Sivakumar, 2000). The (highest resolution) daily rainfall series is contaminated by both measurement noise (due to the influence of wind, wetting, evaporation, gauge exposure, instrumentation and human error in reading the data) and dynamical noise. As 
the 2-day, 4-day and 8-day rainfall series are obtained simply by adding the appropriate number of daily rainfall values, the noise levels in these series relative to the daily series may be interpreted in two possible ways. If the daily series is contaminated by random noise, then the noise levels in the 2-day, 4-day and 8-day series may be almost the same as that in the daily series. However, if the noise is systematic (either underestimation or overestimation of rainfall), then the noise levels in the lower resolution series will be (significantly) higher than that in the daily series. In the absence of any information about the type and level of noise present in the daily rainfall series, any conclusion on the level of noise in the other three series is very difficult to provide. However, as the systematic noise is more dominant (compared to other types of noise) in a tipping bucket raingauge (the type of gauge used for rainfall measurement at the Leaf River basin), it is possible that the 2-day, 4-day and 8-day rainfall series have, in order, increasing noise levels. In view of this, the dimension results obtained for the rainfall series of different scales indicate that the highest dimension is obtained for the 8-day rainfall, which in all probability has the highest noise level, whereas the daily rainfall, probably having the lowest noise level, yields the lowest dimension. These results suggest that overestimation of the dimension of the lower resolution series (due to noise) could be much higher than that of an underestimation of the dimension at higher resolution scales (due to data size).

The problems of data size and noise are encountered in almost every field of natural and physical phenomena, including hydrology (e.g. Havstad and Ehlers, 1989; Schreiber and Kantz, 1996; Sivakumar et al., 1999b). It is important to note, however, that there could also be other problems, as serious as or even more serious than the above, that might not have received the necessary attention because of their association with a particular field. One such problem commonly encountered in the field of hydrology (particularly rainfall) is the presence of a large number of zeros in the measurements. In the presence of a large number of any single value in a time series, the reconstructed hypersurface in phase-space will tend to a point and may result in a significant underestimation of the correlation dimension (e.g. Tsonis et al., 1994).

The statistics of the four rainfall series analysed in the present study (Tables 1 and 2) indicate that the percentage of zeros are 54.53, 39.87, 20.12, and 6.05, for daily, 2-day, 4-day and 8-day rainfall, respectively. As expected, the percentage of zeros in the highest resolution rainfall, i.e. daily, is greater than that in all the other series, and continues to decrease for lower resolution series until it reaches the lowest level for 8-day series. The percentage of zeros in the four series suggests that the dimension of the daily rainfall series could be underestimated significantly and the extent of underestimation decreases for the 2-day and 4-day series until it reaches the lowest level for the 8-day series. The dimension results obtained for the four series support the above. If the elimination of the large number of zeros from the series is possible, then the underestimation of the dimension can be avoided, in which case the highest resolution series may result in the highest dimension; then the change in the dimension could coincide with the change in the coefficient of variation, thus representing the true variability of the dynamics of the series. However, the dimension value of even as high as 8.87 obtained for the 8 day rainfall series (with $6.05 \%$ of zeros) could be an underestimation of the actual dimension, if the influence of noise is not significant in the dimension estimate.

The possible underestimation of the dimension due to the presence of a large number of zeros in the rainfall series may also be explained as follows. Assuming a (daily) rainfall series of dimension $d=4.82$, in all embedding dimensions $m<4.82$, the object is space filling. Thus, for $m<5, n=m$, while for $m^{3} 5, n=4.82$. Thus, the first deviation of the correlation exponent from the diagonal (i.e. $n=4.82$ starting at $m=5$ and remaining constant for higher values of $m$ ) against the embedding dimension should provide an estimate of the correlation dimension. However, for the rainfall series analysed in the present study (and many other natural time series), Fig. 3(b), the plot of the correlation exponent versus embedding dimension, shows that for values of $m<5, n^{1} m$ (but $n<m$ ), and $n=4.82$ not for all values of $m^{3} 5$, but only when the value of $m$ is large (about 13). In fact, the values of $n$ are underestimated consistently for all values of $m$. As a result, the first deviation in the correlation exponent from the diagonal and the saturation of the correlation exponent (i.e. d) do not occur at the same embedding dimension. Instead, the correlation exponent deviates from the diagonal for values of $m$ even less than 5 (e.g. $m$ as low as 1 ) and the deviation continues for higher values of $m$ until saturation. This observation is not consistent with the concept of the phase-space reconstruction, where $n$ must be equal to $m$ for all values of $m<d$. A possible reason for this could be the presence of a large number of a single value (zero in this case) occurring in the series. Figure 3(b) indicates that significant underestimation of the correlation exponent (against $m$ ) occurs for the daily series and the underestimation of $d$ is very small for the 8-day series. Interestingly, the daily rainfall series contains the highest percentage of zeros $(54.53 \%)$ and the 8 -day series contains the lowest percentage of zeros $(6.05 \%)$. A decrease in trend in the dimension estimate is observed also for 2-day and 4day rainfall, which have zero values of $39.87 \%$ and $20.12 \%$ respectively. These observations suggest the extent of the 
influence of the large number of zeros on the correlation dimension estimation (i.e. underestimation)."

In general, the dimension estimation of a time series can be influenced by, but not limited to, data size, noise and percentage of zeros. However, the observations made above seem to indicate that the data size has very little influence on the dimension estimation, whereas the presence of noise and the large number of zeros may have significant effects. Also, recent studies (e.g. Sivakumar et al., 1999b; Sivakumar, 2000) reveal that, while small levels of noise influence significantly the accuracy of prediction estimates, the correlation dimension estimates are not influenced significantly (i.e. overestimated) even when noise levels are high. Hence, a significant part of the difference in the dimensions of the daily and 8-day rainfall series may well be attributed to the underestimation of the dimension due to the presence of zeros in the series. This leads to the suggestion that the dimensions of the higher resolution rainfall series are significantly underestimated.

The above results suggest that the dimensions are influenced more significantly by the presence of a large number of zeros than by noise or data size, particularly in the higher resolution series, such as daily and 2-day, in which more than one-third of the values are zeros. However, most studies of chaos in rainfall series analysed daily or even higher resolution series (that with the exception of storm events, may contain a large number of zeros) without investigating the influence of the zeros. As those studies analysed rainfall series of only one particular scale observed in the same geographical location or of different scales observed in different geographical locations rather than rainfall series of different scales at the same location, the accuracy of the dimension of the series could neither be quantified nor the influence of the zeros investigated. The availability of the coefficient of variation for each of the series analysed in the present study could verify the accuracy of the dimension estimate and the possible influence of zeros (underestimation) as it analysed rainfall series at four different scales. The inverse relationship between the correlation dimension and the coefficient of variation suggests the possible influence of a large number of zeros.

As it is typical to observe a large number of zeros in rainfall series, particularly the higher resolution ones, this observation adds an additional important question about the application of the correlation dimension method to rainfall series, . A zero value in the rainfall series is as important as any other value in the series and, therefore, cannot be eliminated in the correlation dimension estimation. On the other hand, since the lower resolution rainfall series considered in the present study are expected to have significant levels of noise, it may be necessary to study its influence (overestimation) by estimating and subsequently reducing the levels of noise. The outcomes of such studies could be very useful in understanding the above problems and in verifying the present results regarding the correlation dimensions of the rainfall series and the existence of chaos in the dynamics of rainfall transformation between the different scales studied.

\section{Conclusions and scope for further study}

Understanding the dynamical behaviour of transformation between the rainfall processes observed at different scales is important to identify the suitable type of approach and the possibility of transformation of data from one scale to another. Using the correlation dimension method, the present study investigated rainfall series of four different temporal scales, daily, 2-day, 4-day and 8-day, observed at the Leaf River basin, Mississippi, USA. The underlying assumption was that the dynamical behaviour of the rainfall processes at the different scales could provide important information regarding the behaviour of the overall transformation process between these scales.

The fact that the correlation dimension method yielded finite dimension values of 4.82, 5.26, 6.42, and 8.87 for the daily, 2-day, 4-day and 8-day rainfall series respectively suggests that all of the above rainfall series exhibited chaotic behaviour. Hence, the transformation process between these scales might also be chaotic; however, such an interpretation must be further substantiated. Investigation of any parameter that connects the above rainfall series could be useful. The distribution of rainfall from one scale to another is one such important parameter, studies of which might lead to additional information regarding the behaviour of transformation processes at the Leaf River basin.

The correlation dimension results showed that the higher resolution rainfall series yielded lower dimensions than did the lower resolution series, suggesting that the variability of the higher resolution series was less than that of the lower resolution series. Unfortunately, aggregations of rainfall from higher to lower resolutions normally decrease the variability; this suggests that the correlation dimension method may have serious limitations. Verification, using values of the coefficient of variation, revealed that the dimensions of the higher resolution rainfall series might have been underestimated significantly because of the presence of a large number of zeros in these series compared to those in the lower resolution series. On the other hand, the dimensions of the lower resolution series might have been overestimated slightly due to the presence of higher levels 
of noise in these series compared to those in the higher resolution ones. It is important, therefore, to study the problems of the presence of zeros and noise in the rainfall series if realistic dimension estimates and variability of the rainfall series are to be obtained.

Although the present study did not answer concusively the existence of chaotic behaviour in the dynamics of rainfall transformation process between different scales, it has provided some clues so as not to exclude such a possibility and justifies continuation of the investigation to confirm the existence of a chaotic component in the transformation process. In addition to the possible further directions provided above, e.g. analysis of distribution of weights, study of the presence of zeros and noise in the rainfall series, other chaos identification methods, such as the nonlinear prediction method, may support the present results. If confirmed, the presence of chaotic behaviour could provide interesting openings for a better understanding of the rainfall transformation process.

\section{Acknowledgments}

The author would like to thank Dr. Jiri Stehlik and an anonymous reviewer for their valuable suggestions, which resulted in a more accurate and complete presentation of the work.

\section{References}

Berndtsson, R., Jinno, K., Kawamura, A., Olsson, J. and Xu, S., 1994. Dynamical systems theory applied to long-term temperature and precipitation time series. Trends Hydrol., 1, 291-297.

Grassberger, P. and Procaccia, I., 1983. Measuring the strangeness of strange attractors. Physica D, 9, 189-208.

Havstad, J.W. and Ehlers, C.L., 1989. Attractor dimension of nonstationary dynamical systems from small data sets. Phys. Rev. A, 39, 845-853.
Holzfuss, J. and Mayer-Kress, G., 1986. An approach to errorestimation in the application of dimension algorithms. In: Dimensions and Entropies in Chaotic Systems, G. Mayer-Kress, (Ed.). Springer, New York, USA, 114-122.

Jayawardena, A.W. and Lai, F., 1994. Analysis and prediction of chaos in rainfall and stream flow time series. J. Hydrol., 153, 23-52.

Koutsoyiannis, D. and Pachakis, D., 1996. Deterministic chaos versus stochasticity in analysis and modeling of point rainfall series. J. Geophys. Res., 101, 26441-26451.

Krasovskaia, I., Gottschalk, L. and Kundzewicz, Z.W., 1999. Dimensionality of Scandinavian river flow regimes. Hydrolog. Sci. J., 44, 705-723.

Porporato, A. and Ridolfi, L., 1997. Nonlinear analysis of river flow time sequences. Water Resour. Res., 33, 1353-1367.

Puente, C.E. and Obregon, N., 1996. A deterministic geometric representation of temporal rainfall: Results for a storm in Boston. Water Resour. Res., 32, 2825-2839.

Rodriguez-Iturbe, I., De Power, F.B., Sharifi, M.B. and Georgakakos, K.P., 1989. Chaos in rainfall. Water Resour. Res., 25, 1667-1675.

Schreiber, T. and Kantz, H., 1996. Observing and predicting chaotic signals: Is $2 \%$ noise too much? In: Predictability of Complex Dynamical Systems, Yu.A. Kravtsov, and J.B. Kadtke, (Eds.). Springer Series in Synergetics, Springer, Berlin, Germany, 43-65.

Sivakumar, B., 2000. Chaos theory in hydrology: important issues and interpretations. J. Hydrol., 227, 1-20.

Sivakumar, B., Liong, S.Y., Liaw, C.Y. and Phoon, K.K., 1999a. Singapore rainfall behavior: Chaotic? J. Hydrol. Eng., ASCE, 4, 38-48.

Sivakumar, B., Phoon, K.K., Liong, S.Y. and Liaw, C.Y., 1999 b. A systematic approach to noise reduction in hydrological chaotic time series. J. Hydrol., 219, 103-135.

Stehlik, J., 1999. Deterministic chaos in runoff series. J. Hydrol. Hydromech., 47, 271-287.

Takens, F., 1981. Detecting strange attractors in turbulence. In: Dynamical Systems and Turbulence, D.A. Rand, and L.S. Young, (Eds.). Lecture Notes in Mathematics, 898, Springer, Berlin, Germany, 366-381.

Tsonis, A.A., Elsner, J.B. and Georgakakos, K.P., 1993. Estimating the dimension of weather and climate attractors: important issues about the procedure and interpretation. J. Atmos. Sci., 50, 25492555.

Tsonis, A.A., Triantafyllou, G.N., Elsner, J.B., Holdzkom II, J.J. and Kirwan Jr., A.D., 1994. An investigation of the ability of nonlinear methods to infer dynamics from observables. Bull. Amer. Meteorol. Soc., 75, 1623-1633. 
